\title{
Einiges über die periodische Augenentzündung der Pferde.
}

Von

Dr. Albrecht Nagel.

\begin{abstract}
$P_{\text {athologische Beobachtungen am Thierauge sind bei dem }}$ heutigen Stande der Augenheilkunde in hohem Grade geeignet, diese Wissenschaft zu fördern. Nicht allein dass die leichtere Zugänglichkeit des afficirten Auges für anatomische Untersuchungen, namentlich in früheren Stadien der Krankheit, für die Erforschung des Krankheitsprocesses viel zu leisten verspricht; selbst die Therapie wird Vortheil daraus zichen können, dass bei dem kranken Thierauge das Experiment um vieles leichter angewendet werden darf als beim menschlichen Auge. Fs wird daher der Rechtfertigung nicht bedürfen, wenn ich von einigen ersten Resultaten des. Studiums der Augenkrankheiten des Pferdes, welche ich andorwärts ausführlicher theils dargelegt habe*), theils darlegen werde, hier ein kurzes Resumé gebe.
\end{abstract}

*) Magazin für Thierheilkunde Bd. XXIX Ileft I. 
Die sogenannte periodische Augenentzündung oder die Mondblindheit des Pferdes führt ihren Namen nach dem der grossen Mehrzahl der Fälle eigenthümlichen periodenweisen Auftreten, dem intermittirenden oder remittirenden Charakter der Entzündung. In den Perioden wollte Mancher ein Zusammentreffen mit dem Mondwechsel erkennen. Es mangelt aber nicht allein ein wissenschaftlicher, das Wesen bezeichnender Namen der Krankheit; unsre ganze Kenntniss derselben ist bisher noch sehr lückenhaft. Dem, was man vor funfzig Jahren über die periodische Augenentzündung wusste, ist seitdem wenig hinzugefügt worden, namentlich steht die Behandlung noch durchaus auf demselben Standpunkt wie damals, $d . h$. die Krankheit wird so gut wie allgemein als eine unheilbare, mit Erblindung endigende, betrachtet, die höchstens in ganz seltenen Fällen einmal spontan, ohne alles Zuthun der Kunst einen günstigen Ausgang nimmt. Mehr Aufmerksamkeit als der Therapie hat man der Aetiologie der Krankheit gewidmet, doch auch hier ist man noch weit davon entfernt, sichere, allgemein anerkannte Resultate zu haben. Ohne hier auf dies weitschichtige, practisch sehr wichtige Thema einzugehen, bemerke ich nur, dass ich Grund habe, zu glauben, dass es bei dem verschiedenen epizootischen und enzootischen Vorkommen der periodischen Augenentzündung sich um verschiedene Varietäten des Leidens handele. Von grossem Interesse ist übrigens das Zusammentreffen des epizootischen Auftretens mit anderweiten Epizootien und Epidemieen. Namentlich ist das gleichzeitige Ersicheinen periodischer Ophthalmie der Pferde mit granulöser Bindehautentzündung des Menschen und mit typhösen Erkrankungen constatirt.

Ueber die Natur der periodischen Augenentzündung hat man viel gestritten, noch die neuesten Autoren sind darüber nicht einig. Während van Biervliet und 
van $\mathrm{Rooy}^{*}$ ) die Analogie mit dem Glaucom des Menschen hervorheben und darauf ihre therapeutischen Vorschläge basiren, betont Sichel**) die Unterschiede beider Krankheiten und bezeichnet die periodische Augenentzündung als Iridochorioiditis.

Nach den Ergebnissen des anatomischen Messers am todten Auge und den ophthalmoskopischen Befunden am lebenden Auge, welche letzteren ich in reichlicher Anzahl gewonnen habe, wird es nunmehr möglich sein, mit grösserer Sicherheit die Frage zu entscheiden. Und die Entscheidung wird nach meinen Erfahrungen lauten: Beide Theile haben in gewisser Weise Recht, in ihrer Exclusivität aber ist keine von beiden Ansichten gerechtfertigt. Der Name periodische Augenentzündung ist eine gemeinsame, wenig wissenschaftliche Bezeichnung für verschiedenartige Processe. Die Iridochorioiditis und Iridocyclitis mit vorwiegend plastischem Exsudat ist diejenige Form, welche am häufigsten vorkommt. Doch es ist keineswegs die einzige. Es kann - und das ist durchaus nicht selten eine reine Iritis vorkoummen, welche in ihren Symptomen gänzlich mit der sogenannnten periodischen Augenentzündung zusammenfällt und späterhin, namentlich bei ungenügender Therapie, oft in Iridochorioiditis übergeht. Zuweilen nimmt die gewöhnlich nur in geringem Grade afficirte Hornhaut in hervorragender Weise an dem Entzündungsprocesse Theil, und man muss die Krankheitsform als Kerato-Iritis bezeichnen. Seltener scheint reine Chorioiditis vorzukommen, bei der die Iris anfangs nicht merkbar mitleidet, dagegen gestaltet sich das Bild der Iridochorioiditis verschieden, je nach der vorherrschenden Betheiligung der lris, des Ciliarkörpers oder der

*) Annales d'oculislique 1861 pag. 125.

**) Ibidem pag. 181. 
Choroidea. Endlich kommen entschieden glaukomatöse Entzüydungsformen (acute und chronische) vor, mit Steigerung des intraocularen Drucks, welche auch in ihrem Verlauf und ihren Ausgängen von den oben genannten Formen ganz verschieden sind. Die höchsten Grade der Krankheit nehmen zuweilen einen schnellen Uebergang in Panophthalmitis.

Zur Begründung dieser Behauptungen führe ich folgende Thatsachen an. Erscheinungen der Iritis kommen, der grossen Mehrzahl von Fällen der sogenannten periodischen Augenentzündung, und zwar vorzugsweise beim Beginn zu: Verengerung der Pupille, Exsudation im Gcwebe der Iris, in der vorderen Kammer, an der hinteren Wand der Hornhaut (Beschläge der Descemet'schen Haut), auf der vorderen Kapsel, Verlöthungen der Iris mit der letzteren (die beiden letzteren mit Unrecht von manchen Thierärzten als abgelöste Traubenkörner bezcichnet). Dic hinteren Synechien sind es, welche in vielen Fällen die chronische Fortdauer der Krankheit und die Recidive der Entzündung verursachen. Eirscheinungen von Keratitis treten häufig zur Iritis hinzu.

Die heftigere Betheiligung des Ciliarkörpers an der lintzündung glaubte ich in den Fällen annehmen zu müssen, wo bei wenig sichtbarer Affection der Iris sehr viel flockiges Exsudat in der vorderen Kammer flottirte oder sich zum Hypopyon gestaltete und, wo ähnlich wie beim sogenannten Hypopyon posticum des Menschen, gelbliche membranartige Exsudate im vordersten Theile des Glaskörpers sichtbar wurden.

Die directe ophthalmoskopische Beobachtung der Choroidea im Entzündungsanfall ist beim Pferde mit so grossen Schwierigkeiten verknüpft, dass sie für die Diagnose oft nicht $\mathrm{zu}$ verwerthen sein wird. Viel leichter aber lassen sich die Folgen der Entzündung der Choroidea in den Vcränderungen des Glaskörpers nachweisen. 
Vertlüssigung des Glaskörpers mit beweglichen Trübungen mannigfaltiger Art habe ich mittelst des Augenspiegels zuweilen im Entzündungsfall selbst festgestellt; bei weitem öfter aber in den Zwischenzeiten zwischen den acuten Perioden, wenn sich der Inhalt der vorderen Kaminer wieder aufgehellt hat und nun die ophthalmoskopische Untersuchung der tieferen Theile des Auges möglich wird. Die Abnormitäten des Glaskörper's sieht man oft fortdauern, wenn die Iris wieder gank gesund und beweglich erscheint. Oft sind sic dic einzigen, bloss vermittels des Augenspiegels wahrnehmbaren Zeichen vorangegangener Erkrankung, wenn sonst das Auge bei genauester Untersuchung keincrlei Abweichung vom gesunden Zustande zeigt. Zuweilen ist eine leichte Erweiterung und Schwerbeweglichkeit der Pupille - relativ zu der des andern, gesunden Auges - damit vergesellschaftet. Die Diagnose dieser, wenn auch ganz leichten Glaskörpertrübung ist von um so grösserer practischer Wichtigkeit, als sie die Prognose trotz der anscheinenden Unbedeutendheit um ein Wesentliches verschlechtert. Zuweilen kann man hienach bei einem anscheinend vollkommen gesunden Auge die Rückkehr der Entzündung mit endlichem Ausgang in Netzhautablösung und Cataract vorbersagen.

Eine von der beschriebenen sehr verschiedene, übrigens aber durchaus typische Krankheitsform ist die folgende, welche ich als glaukomatös auffasse: Nach kurzen Prodromen allgemein entzündlicher Erscheinungen, die gewöhnlich den Charakter beginnender Iritis zeigen, manchmal mit allgemeiner Keratitis verbunden, stellt sich eine deutliche Erweiterung der vorhin verengerten Pupille ein. Der Inhalt der vorderen Kammer ist trübe, aber mehr allgemein und gleichmässig, als durch Exsudattlocken. Bei der Paracentese der Hornhaut fand ich den Humor aqueus dunkel weingelb, aber durchsichtig. 
Charakteristisch ist num aber, dass die zuweilen etwas unregelmässig dilatirte P'upille cine schr auffillend schmutzig dunkelgrüne gleichmässige Färbung zeigt, mitunter von cinem fast metallischen Gilanze begleitet. Jan muss diese äusserst charakteristische Färbung, welche vicle V'eterinäre als grünen Staar bezeichnen, (womit natürlich gar nichts über die Natur, Sitz, Bedeutung des Zustan(les gesagt ist), nicht mit andern grünlichen Färbungen der Pupille verwechseln. Die Pupille des gesunden Pferrleauges, namentlich die dilatirte, zeigt eine rauchgrauc, bis grünliche Färbung, die bei geeigneter lieleuchtung in einen schönen, hell gelblich grünen Glanz übergeht und lediglich durch das 'Tapetum der Choroidca bedingt ist. Andererseits zeigen die meisten an Iritis und Iridochorioiditis erkrankten Augen einen grünlichen Schimmer, indem die Exsulate in der vorderen Kammer und im Glaskörper von weisslicher, grauer, gelblicher Färbung im Verein mit dem vom Augengrunde reflectirten licht eine solche unbostimmte grünlich graue Verfärbung hervorbringen. Diese grünliche Verfärbung der Pupille wird von manchen für charakteristisch für die periodische Augenentzündung überhaupt angesehen, z. B. ganz neuerdings von Gerlach"), jedoch, wie ich glaube, mit Unrecht. Es hängt ganz von der Yenge und Beschaffenheit der Exsudate ab, ist rein zufällig und wechselt im Verlauf der Krankheit öfters, ob die Färbung eine weisslich grane oder gelbliche oder grünliche ist. Ganż auffallend und eigenthümlich ist hingegen das Aussehen der I'upille bei der glaukomatösen Entzündungsform, übrigens durchaus ähnlich der bei den gleichen Ieiden des menschlichen Auges vorkommenden Färbung, welche die Entstehung des Namens veranlasst hat $(\gamma \lambda \omega v x \grave{s})$. Die dunkelgrünc liärbung ist auch von der

*) Handbuch der gerichtlichen Thierheilkunde. 1862. 
natürlichen Farbe des von der Tapetumschicht reflectirten Lichtes gänzlich verschieden. Die Ursache derselben suche ich in einer Auflösung des Blutfarbstoffes und Beimischung dadurch gefärbten Exsudates in den Medien des Auges, dem Humor aqueus und vitreus. Hätte man Gelegenheit, die Farbe des Glaskörpers in solchen Fällen zu untersuchen, so würde man, glaube ich, eine ebenso weingelbe bis röthliche Verfärbung desselben finden, wie ich sie beim Kammerwasser constatirt habe. Diese zusammen mit der natürlichen Färbung des Tapetum giebt die schmutzige Mischfarbe, in welcher das Dunkelgrün vorherrscht. Die Untersuchung mit dem Augenspiegel ergiebt in solchen Fällen, dass die Details des Augengrundes durchaus nicht erkennbar sind, die Pupille erscheint schwach beleuchtet, in einem gleichmässig trüben, schmutzig röthlichem Licht und in derselben sieht man oft ein staub- oder schleimartiges, graues, bewegliches Wesen.

Man wird hier die Analogie mit den ophthalmoskopischen Erscheinungen bei dem acuten Glaukom des Meuschen im Entzündungsanfalle nicht verkennen. Ich füge hinzu, dass auffallende Zunahme der Consistenz des Bulbus, Verflachung der vorderen Kammer und mehr oder weniger vollständige Amaurose das Bild des Glaukoms vervollständigen. Es scheint unzweifelhaft, dass der vermehrte intraoculare Druck lähmend auf die Netzhaut und auf die im Stroma der Choroidea verlaufenden Nerven der Iris und Hornhaut einwirken.

Zwischen den erwähnten typischen Formen giebt es nun Uebergänge, Mischformen, die bald nach der einen, bald nach der andern Seite neigen und deren Prognose und Behandlung demzufolge verschieden ist. Die Nothwendigkeit der Individualisirung tritt, wie überall im Gebiete der practischen Medicin, so auch hier hervor.

Ueber den Verlauf und die Ausgänge der verschie- 
denen Krankheitsformen sei nur so vicl gesagt, dass die Analogie mit den Vorgängen im menschlichen Auge unter entsprechenden Umständen eine sehr vollständige zu sein scheint. Zur Iritis, die oft von Hornhautaffection begleitet ist, gesellt sich im weiteren Verlauf, nachdem sich hintere Synechien gebildet haben, fast inmer Mitleidenschaft der Choroidea. Die Irido-Chorioiditis führt dann bei längerem Bestehen unter zunehmender Frweichung und Trübung des Glaskörpers zur Ablösung der Netzhaut durch subretinale serösc Ergüsse und nach dieser und auch ohne diese zur Staarbildung, am letzten Iinde aber zur Phthisis bulbi. Bald bilden sich zuerst kleine Staarpunkte und Flecken, von der Grösse einer Nadelspitze bis zur Grösse eines Stecknadelkopfes und darüber, bald entsteht ohne solche abgegrenzte Trübungen eine allgemeine Trübung des Linsensystems. Die Entstehung der partiellen Linsentrübungen hängt oft noch weiter mit der Ablagerung iritischer Exsudate auf der vorderen Kapsel zusammen.

Ueber die Natur und Entstehung der sog. Staarpunkte, welche in solcher Weise im menschlichen Auge nicht beobachtet werden, fehlt es noch an genügenden Untersuchungen. Dieselben bilden sehr feine inselartigc Trübungen an der vorderen Oberfläche der linse meist von geringer Intensität, so dass sie viel Licht durchlassen. Auf dem hell erleuchteten Augengrunde markiren sie sich daher selır wenig und werden bei ophthalmoskopischen Untersuchungen leicht übersehen. Viel leichter entdeckt man sie mit freiem Auge bei gewöhnlicher auf einer Seite coupirter Beleuchtung oder bei Focalbeleuchtung. Ohne Zweifel haben diese Trübungen, welche gewöhnlich stationär bleiben und keine Cataractbildung nach sich ziehen, ihren Sitz in der die innere Kapselfläche bekleidenden Zellschicht, und ich trage kein Bedenken, sie direct als Entzündungsproduct anzusprechen. 
Man sieht Staarpunkte zuweilen in wenigen Tagen entstehen, nach dem Entzündungsanfall zurückbleiben. Die Linsenkapselentzündung spielt dieselbe Rolle wie die Entzündung der Descemet'schen Membran, weIche ja auch im menschlichen Auge die constante Begleiterin mancher Formen von Iritis ist.

Auf ganz antere Weise geschieht die Erblindung beim Glaukom. Hier kann völliger oder fast völliger Verlust der Sehkraft stattfinden, ehe noch die Durchsichtigkeit der Linse sich merkbar vermindert hat und wo auch die Trübung in den übrigen Medien des Auges in kcinem Verhältniss zu der Abnahme des Sehvermögens steht. Es ist das eine Amaurose, die unstreitig durch die Compression der Netzhaut und des Sehnerven begründet ist. Sie kann sofort aufhören, wenn das die Compression bedingende Moment ans dem Wege geräumt wird. Excavation des Nehnerven glaube ich einige Male wahrgenommen zu haben, doch will ich die Entscheidung darüber noch dahin gestellt sein lassen, um so mehr, da die Beurtheilung des Profils der Sehnervenpapille beim Pferde durch den Mangel grosser Gefässstämme erschwert wird, deren charakteristisch veränderter Verlauf doch beim Menschen am meisten das Urtheil über das Niveau der Papille leitet. Bcim Pferde lassen sich hekanntlich Centralgefässstämme (Arterien und Venen) nicht wie beim Menschen unterscheiden, sondern eine Anzahl feiner Gefässchen verbreitet sich divergirend in die Retina. Auch die glaukomatösen Entzündungsformeu pflegen zur Cataractbildung und schliesslich zur Atrophie des Bulbus zu führen, und wenn der glaukomatöse Staar auch in manchen Fällen besondere Eigenthümlichkeiten bewahrt, so confluiren in der letzten Periode die Ausgänge aller Formen doch so schr, dass über die lintwicklungsweise sich oft kein Urtheil fällen lässt.

$\mathrm{Zu}$ interessanten Resultaten bin ich bei meinen 
Untersuchungen über die Therapic der verschiedenen Species periodischer Augenentzündung gelangt. Die Anwendung der in der menschlichen Augenheilkunde geltenden therapeutischen Grundsätze hat sich mir zum grossen Theile als ausführbar und nutzenbringend erwiesen. Mit denselben Mitteln, welche bei Keratitis, Iritis, Iridochorioiditis, Glaukom des Menschen benutzt worden, habe ich beim Pferde dic erfreulichsten Resultate erreicht.

Bei einfacher exsudativer Iritis ist das schwefelsaure Atropin das souveraine Heilmittel. Der consequente methodische Gebrauch dessclben hat hier namentilich in der frühesten Periode der Krankheit, man kann sagen, eine specifische Wirkung. Die Rulıstellung der Muskelfasern der Iris, die Verminderung der IIyperämie, die Herabsetzung des intraocularen Druckes wirken zusammen zur Sistirung der Entzündung. Die Dilatation der Pupille hindert auf rein mechanische Weise das Absetzen fester Lxsudate in's Pupillargebiet und dic Anlöthung der Iris an die Linsenkapsel, und damit fällt ein wesentliches ursächliches Moment für dic Recidive dcr lintzündung fort. lis entstehen keine hinteren Synechien, welche durch das immerwährende Hemmniss, welehes sic dem freien Spiel der Pupille entgegensetzen, permanent reizend wirken. Viel kommt darauf an, die Anwendung des $\Lambda$ tropin recht zeitig vorzunehmen, damn ist die Wirkung an präcisesten und schnellsten. Die volle Lintwicklung des Entzündungsanfalls wird verhindert, oft der Anfall im ersten Lintstehen coupirt. Erncuert sich dic Entzündung der Iris einmal oder öfter, so lässt sich das Mydriaticum oft mit gleichem Erfolge anwenden.

Wo das schwefelsaure Atropin nicht allein die Entzündung zu brechen und die verderblichen Folgen zu beseitigen vermag, da bildet es ein wichtiges Lnterstützungs- 
mittel für anderweitige Heilagentien. Ich hebe hier nur die wichtigsten hervor und diese sind operative.

Zuerst die Paracentese der vorderen Kammer. Der Indicationen für dieselbe giebt es mehrere. Zuvörderst starke Entzündung der Hornhaut, so dass diese für dic Atropinlösung nicht genügend permeabel ist; sodann massenhafte Exsudate in der vorderen Kammer, besonders bei flockigem und eitrigem Exsudate, also bei Hypopyon; ferner bei exsudativen Beschlägen auf der hinteren Hornhautwand, wo die Descemet'sche Membran mit zahlreichen Pünktchen übersät erscheint; endlich, wo die intraoculare Spannung in nicht zu hohem Grade gesteigert ist. Einmalige oder mehrmalige Anwendung der Punction beseitigt die heftigen und gefahrdrohenden Erscheinungen. Eine etwaige Hornhautentzündung nimmt einen viel schnelleren und günstigeren Verlauf, die Exsudate werden aus der vorderen Kammer entleert und bilden sich entweder gar nicht von Neuem, oder doch in viel geringerem Maasse; Fxsudate von der Descemet'schen Haut und der vorderen Linsenkapsel, wenn sle noch ganz frisch sind, verschwinden oft nach Entleerung des Kammerwassers; die zu grosse Spannung im Auge mit ihren uachtheiligen Folgen wird wenigstens für eine kurze Zeit herabgesetzt. Oft ist die Paracentese das Mittel, der Einwirkung des Mydriaticums den Weg zu bahnen. Beiläufig bemerke ich hier, dass ich auch bei traumatischen Ophthalmien des Pferdes die vortheilhafteste Wirkung von der Paracentese der Hornhaut beobachtet habe, ja dies waren die ersten Fälle, in denen ich vor mehreren Jahren die Operation versuchte. In Folge von Verletzungen war heftige Kerato - Iritis mit Abscessbildung in der Hornhaut eingetreten. Die ganze Hornhaut war trübe, die Hälfte der Hornhaut und darüber in der Abscessbildung begriffen, es drohte die Per- 
foration. Auf die Analogie beim Menschenauge gestätzt, griff ich, den sicheren Ruin des Auges vor mir sehend, zur Paracentese der Hornhaut und das Auge wurde dadurch gerettet. Gleich nach der ersten Punction geschah dem Umsichgreifen der Abscedirung Einhalt, nach mehreren weiteren Punctionen, dic in Zwischenräumen von 24 Stunden ausgeführt wurden, hatte die Hornhaut ein unvergleichlich besseres Aussehen, die Prominenz des Abscesses war geschwunden. Späterhin blieb an der Stelle des Hornhautabscesses eine nicht zu grosse Narbe zurück, welche dem Sehen keinen Eintrag that. Solcher Beobachtungen hatte ich mehrere, gleich günstige. Die Ausführung der Operation anlangend, so habe ich dieselbe in einer grossen Anzahl von Fällen ohne Anwendung des Chloroforms vollzogen nnd niemals nachtheilige Reaction gesehen. Die störende Thätigkeit des musculus pyramidalis, welcher das Auge mit grosser Kraft in die Orbita zurückzieht, ermüdete ich durch öfteres Berühren des Bulbus und benutzte dann einen Moment der Ruhe zur Ausführung der Operation. Will man jedoch, und dazu wird sich zuweilen Gelegenheit bieten, eine Loslösung nicht zu alter hinterer Synechien mit der Punction verbinden, so ist die Anwendung des Anästheticums nicht $\mathrm{zu}$ entbehren.

Endlich thue ich noch Erwähnung eines Hauptmittels für gewisse vorgeschrittnere Fälle, der Iridectomie.") Die Indicationen für diese Operation fasse ich nach meinen bisherigen Erfahrungen dahin zusammen: Breite Adhäsionen der Iris an die vordere Kapsel oder totale hin-

*) Der Vorsehlag, bei periodischer Ophthalmie die Iridectomie zu machen, ist, wie mir nachträglich bekannt wurde, bereits von Didot, Annal. de méd. vétérinaire de Bruxelles 1860, so wie von ya n Bier vliet und van Rooy in der oben citirten Arbeit gemacht worden, doch fehlte diesen Autoren die Gelegenheit zur Ausfïhrung. 
tere Synechie bei noch nicht oder doch noch nur sehr unbedeutend getrübter Linse. Ferner - selbst schmale Synechien, wenn sie durch ihre Lage besonders nachtheilig (zerrend) einwirken und einen chronischen Entzündungszustand nähren. Sodann sehr starke dauernde Verengerung der Pupille, die sich durch Atropin und Punction schlechterdings nicht heben lässt und endlich vorzugsweise offenbare pathologische Zunahme des intraocularen Druckes, wie in den Fällen von Glaukom mit Lähmung der Pupille.

Die Iridectomie scheint selbst in schlimmen Fällen noch dem Processe Einhalt thun zu können. Das vorhandene Sehvermögen bleibt, verbessert sich sogar zuweilen bedeutend, die Entzündungssymptome schwinden, mindestens wird in vielen Fällen der gänzlichen Erblindung vorgebeugt. Wirklich frappant ist die Wirkung der Irisexcision bei acuter glaukomatöser Entzündung. Wo schon völlige Amaurose eingetreten ist, aber nicht zu lange gedauert hat, kann diese durch die Operation fast plötzlich aufgehoben werden und die Entzündung kehrt nicht wieder.

Ich habe die Iridectomie beim Pferde stets unter Chloroformmarkose gemacht und mich bei derselben ähnlicher Instrumente bedient, wie sie bei den gleichen Operationen am menschlichen Auge gebräuchlich sind. Nachtheilige Reaction habe ich bis jetat nicht erfolgen schen; meistens trat nicht cinmal Thränen des Auges und Lichtscheu nach der Operation ein.

Dies sind die Heilnittel, von denen ich günstige Lrfolge in einer Reihe von Fällen periodischer Augenentzündung mit zweifelloser Sicherheit besbachtet habe, deren Wirksamkeit ich mithin als festgestellt betrachte. Hieran reihen sich noch manche Mittel, die für die Folge noch $z u$ versuchen sein dürften. Vor Allem wird es darauf ankommen, für gewisse Fälle, welche bis jetzt die 
schlechteste Prognose geben, noch eine wirksamere Therapie aufufinden. Es sind dies Fälle oder Stadien von Fällen, wo Erweichung des Glaskörpers eintritt und sich die Netzhaut durch Ergüsse zwischen sie und die Choroidea abzulösen beginnt. Was hier operativ vielleicht mit Aussicht auf Erfolg zu versuchen wäre, ist die Punction des Glaskörpers und die Perforation der losgelösten Netzhautpartie, wenn die letztere sich ophthalmoskopisch erkennen lässt und eine für diesen Eingriff günstige Lage hat. Bei dem erkrankten Pferdeauge wird man sich zu diesem freilich riskanten Verfahren viel leichter entschliessen mögen, als bei dem gleichen Leiden des menschlichen Auges, und es ist daher von dieser Seite auch für die menschliche Ophthalmologie Nutzen zu erwarten. Ich habe die Absicht, bei den nächsten sich mir darbietenden geeigneten Fällen beim Pferde die erwähnte Operation als ultimum refugium anzuwenden. 